\title{
Rechtsprechung
}

\section{Die Zulässigkeit der Ausfuhr antiker Münzen}

Bundesfinanzhof, Urteil vom 11. Dezember 2012 - VII R 33/11

Archäologische Gegenstände im Sinne der Verordnung (EG) über die Ausfuhr von Kulturgütern (VO Nr. 116/2009) sind nur solche, die einen Wert für die Archäologie haben, also von Menschenhand geschaffene oder bearbeitete Gegenstände, die Erkenntnisse über vergangene Kulturen zu vermitteln vermögen, insbesondere etwa über deren Gebräuche, den damaligen technischen und künstlerischen Entwicklungsstand, politische und gesellschaftliche Strukturen, die Religion und dergleichen mehr. Gegenstände, die anderweit gewonnene Erkenntnisse über vergangene Kulturen allenfalls illustrieren und deshalb für die Archäologie keine Bedeutung haben, sind keine „archäologischen Gegenstände“ oder Funde. Antike Münzen können archäologische Gegenstände sein. (Amtlicher Leitsatz)

\section{Gründe}

\section{I.}

- Der Kläger, Revisionskläger und Revisionsbeklagte (Kläger) hat am 31. Oktober 2008 bei dem Zollamt (ZA) des Beklagten, Revisionsbeklagten und Revisionsklägers (Hauptzollamt - HZA) eine Ausfuhranmeldung über 32 Münzen und Medaillen abgegeben, die - wie in der Anlage zur Ausfuhranmeldung erläutert wurde -1.500 bis 2.400 Jahre alt und zwischen 50 Euro und 400 Euro wert sein sollen. Das ZA hat die Annahme dieser Anmeldung abgelehnt, weil es sich um Kulturgüter iSd Art. 1, Anh. A Nr. 1 der Verordnung (EWG) Nr. 3911/92 des Rates vom 9. Dezember 1992 über die Ausfuhr von Kulturgütern (Amtsblatt der Europäischen Gemeinschaften Nr. L 395/1; im Folgenden: VO Nr. 3911/92) handele, für die eine Ausfuhrgenehmigung vorgelegt werden müsse.

Auf die gegen diesen Bescheid erhobene Klage hat das Finanzgericht (FG) unter Abweisung derselben im Übrigen das HZA mit in der Zeitschrift für Zölle und Verbrauchsteuern 2012, Beilage 2, 19 veröffentlichtem Urteil verpflichtet, den Kläger erneut zu bescheiden. Es ist der Auffassung, bei den Ausfuhrwaren handele es sich zwar um Kulturgüter im Sinne der VO Nr. 3911/92 bzw. der insofern seit dem 2. März 2009 anzuwendenden Verordnung (EG) Nr. 116/2009 des Rates vom 18. Dezember 2008 über die Ausfuhr von Kulturgütern (Amtsblatt der Europäischen Union Nr. L 39/1; im Folgenden: VO Nr. 116/2009), sodass der Kläger die Annahme der Ausfuhranmeldung ohne Vorlage einer Ausfuhrgenehmigung nicht verlangen könne. Art. 2 Abs. 2 Unterabs. 2 VO Nr. 116/2009 räume den Mitgliedstaaten jedoch Ermessen ein, Ausnahmen von der generellen Ausfuhrgenehmigungspflicht zu machen. Dieses Ermessen habe das HZA bisher nicht ausgeübt.

Gegen dieses Urteil richten sich die Revisionen des Klägers sowie des HZA, welche die Verletzung materiellen Rechts rügen.
Der Kläger meint - zusammengefasst -, die VO Nr. 116/2009 bestimme nur den Rahmen, innerhalb dessen die Mitgliedstaaten Kulturgüter bestimmen könnten. Dazu würden in Deutschland nach § 1 des Gesetzes zum Schutz deutschen Kulturgutes gegen Abwanderung (KultgSchG) Verzeichnisse national wertvollen Kulturgutes aufgestellt und veröffentlicht. Darüber hinaus würden durch jenes Gesetz nur Kulturgüter geschützt, die unrechtmäßig auf deutsches Zollgebiet gelangt seien. Zusammen mit dem Kulturgüterrückgabegesetz (BGBI I 2007, 757, 2547) sei insofern ein geschlossenes Überwachungs- und Rückholsystem geschaffen worden.

Einzelne Münzen stünden nicht unter dem Schutz der VO Nr. 116/2009, wie deren Anh. I.A Nr. 13 Buchst. b zeige, in der nur Sammlungen von Münzen als Schutzobjekt genannt seien. Auch seien antike Münzen im Allgemeinen keine archäologischen Gegenstände, sondern in großer Zahl im Handel präsentes Massengut und in zahlreichen Katalogen mit Preisangaben aufgeführt; sie seien erst recht kein nationales Kulturgut, das - und nicht etwa das Interesse an archäologischer Betätigung und Forschung - zu schützen die Union allein eine Kompetenz in Anspruch nehmen könne. Die vom HZA und vom FG für richtig gehaltene Auslegung der VO Nr. 116/2009 brächte im Übrigen den Münzhandel mit drittländischen Kunden in einer mit der Warenverkehrsfreiheit nicht vereinbaren Weise zum Erliegen. Die Behörden wären völlig überfordert, wenn sie für jede Münze die Erteilung einer Ausfuhrgenehmigung bzw. die vom FG geforderte Ermessensentscheidung treffen müssten.

Im Übrigen ist der Kläger der Auffassung, Art. 2 Unterabs. 2 VO Nr. 116/2009 gelte - anders als das HZA meint - unmittelbar und bedürfe keiner Umsetzung durch einen nationalen Rechtsakt. Das ergebe sich aus dem Wortlaut, in dem nicht darauf verwiesen werde, dass die Ermächtigung durch Rechtssatz umzusetzen sei. Stünde die Umsetzung im Belieben der Mitgliedstaaten, käme es überdies zu einer mit Art. 36 Satz 2 des Vertrags über die Arbeitsweise der Europäischen Union nicht 
vereinbaren Diskriminierung der Münzhändler. Für den Schutz von Objekten archäologisch und wissenschaftlich beschränkten Werts fehle es auch an einer unionsrechtlichen Kompetenz. Der Verhältnismäßigkeitsgrundsatz gebiete, solche Objekte vom Schutz auszunehmen, denn sie könnten definitionsgemäß nicht zu den Kulturgütern eines Mitgliedstaats gehören.

Das HZA trägt vor, Art. 2 Abs. 2 Unterabs. 2 VO Nr. 116/2009 enthalte lediglich eine Ermächtigungsgrundlage für die Mitgliedstaaten, eine Ausnahmeregelung durch Rechtsvorschrift zu schaffen. Dies müsse in Deutschland durch Gesetz erfolgen. Ein solches Gesetz gebe es ebenso wenig wie eine diesbezügliche Verwaltungsvorschrift oder -übung. In Deutschland bestehe folglich derzeit keine Möglichkeit, Kulturgüter ohne Ausfuhrgenehmigung auszuführen. Dies sei nach dem Unionsrecht auch nicht zu beanstanden.

\section{II.}

Die Revision des Klägers ist begründet und führt zur dem Klageantrag entsprechenden Verpflichtung des HZA (§ 126 Abs. 3 Satz 1 Nr. 1 der Finanzgerichtsordnung - FGO). Soweit das Urteil des FG das HZA zu einer Ermessensentscheidung verpflichtet hat und sich das HZA dagegen mit seiner Revision wendet, führt vorgenannter, über die Verpflichtung zu erneuter Ermessensentscheidung hinausgehender und diese verdrängender Ausspruch zur Erledigung des betreffenden Klagebegehrens und der Revision des HZA.

Das Urteil des FG verletzt Bundesrecht (§ 118 Abs. 1 FGO).

Nach Art. 2 Abs. 1 der hier bereits anzuwendenden VO Nr. $116 / 2009$, die in den maßgeblichen Bestimmungen mit den Vorschriften der VO Nr. 3911/92 übereinstimmt, dürfen Kulturgüter aus dem Zollgebiet der Gemeinschaft (jetzt: Union) nur ausgeführt werden, wenn eine Ausfuhrgenehmigung vorliegt. Als Kulturgüter gelten nach Art. 1 der Verordnung die in ihrem Anhang I aufgeführten Güter, mithin u.a. mehr als 100 Jahre alte archäologische Gegenstände aus Grabungen und archäologischen Funden zu Lande oder unter Wasser, archäologischen Stätten und archäologischen Sammlungen. Wird von dem Zollbeteiligten eine solche Ausfuhrgenehmigung für die von ihm gestellten Objekte nicht vorgelegt, obwohl es sich um Kulturgüter im Sinne der VO Nr. 116/2009 handelt, kann er nicht nach Art. 63, 62 Abs. 2 des Zollkodex verlangen, dass das HZA seine Zollanmeldung annimmt.

Die Auffassung des FG, dass Art. 2 Abs. 1 VO Nr. 116/2009 im Streitfall greife, verletzt jedoch Bundesrecht. Denn das FG ist von einem unzutreffenden Begriff des „archäologischen Gegenstandes" bzw. „archäologischen Fundes" ausgegangen.

1. Allerdings vermag der erkennende Senat nicht die - von dem Kläger in der mündlichen Verhandlung auch nicht mehr vorgetragene - Auffassung zu teilen, einer Ausfuhrgenehmigung bedürfe es nur für Gegenstände, die gemäß $§ 1$ Kultg-
SchG in das Verzeichnis national wertvollen Kulturgutes aufgenommen sind, nur solche Gegenstände seien also Kulturgut im Sinne der vorgenannten Unionsverordnung und nur für solche Gegenstände könne die Union überhaupt die Kompetenz in Anspruch nehmen, bei ihrer Ausfuhr eine besondere Genehmigung zu verlangen. Eine solche enge Auslegung des Anwendungsbereichs der Verordnung wäre mit deren Zweck nicht zu vereinbaren, Verfahrensregularien für einen in der Union einheitlichen Schutz vor einer Ausfuhr von den Mitgliedstaaten als schützenswert angesehener Gegenstände aufzustellen. Dabei liegt es auf der Hand, dass die Zollstelle in der Regel nicht beurteilen kann, ob ein ihr zur Abfertigung gestellter Gegenstand von einem der Mitgliedstaaten als Kulturgut unter Schutz gestellt worden ist. Dies zu prüfen muss deshalb, wie es die Verordnung vorschreibt, einer dafür von dem betreffenden Mitgliedstaat zu bestimmenden fachkundigen Behörde im Zusammenwirken mit den entsprechenden Behörden der anderen Mitgliedstaaten (vgl. Art. 6 VO Nr. 116/2009) überlassen bleiben und das Ergebnis der Prüfung in deren Ausfuhrgenehmigung zum Ausdruck gebracht werden. Dass ein Gegenstand von einem Mitgliedstaat nach seinen Rechtsvorschriften unter Schutz gestellt worden ist, ist also nicht Voraussetzung für das Erfordernis der Vorlage einer Ausfuhrgenehmigung bei der Ausfuhr aus dem Zollgebiet der Union, sondern nach Art. 2 Abs. 2 Unterabs. 3 VO Nr. 116/2009 Maßstab für die Erteilung der Ausfuhrgenehmigung.

Ob Gegenstände im Sinne vorgenannter Vorschriften "archäologische Gegenstände" sind, lässt sich indes, anders als das FG offenbar meint, nicht allein anhand ihres Alters und ihrer (vom FG auch nur als wahrscheinlich angesehenen bzw. aufgrund eines zweifelhaften Beweises des ersten Anscheins unterstellten) Herkunft aus Funden oder Grabungen beurteilen. Ein archäologischer Gegenstand ist vielmehr nur ein solcher, der einen Wert für die Archäologie hat, also ein von Menschenhand geschaffener oder bearbeiteter Gegenstand, der Erkenntnisse über vergangene Kulturen zu vermitteln vermag, insbesondere etwa über deren Gebräuche, den damaligen technischen und künstlerischen Entwicklungsstand, politische und gesellschaftliche Strukturen, die Religion und dergleichen mehr. Gegenstände, die anderweit gewonnene Erkenntnisse über vergangene Kulturen allenfalls illustrieren und deshalb für die Archäologie keine Bedeutung haben, sind keine „archäologischen Gegenstände" oder Funde iSd Anh. I VO Nr. 116/2009. Dem entsprechen die Erläuterungen zum Harmonisierten System betreffend die Position 9705, auf welche der vorgenannte Anhang Bezug nimmt, und die ebenfalls auf das "archäologische Interesse" abstellen, welches sie u.a. zum Studium früherer Generationen geeigneten Gegenständen beimessen, bei deren beispielhafter Aufzählung sie übrigens Münzen und Medaillen nicht erwähnen. Dem entspricht es ferner, dass Gegenstände, die für die Archäologie keinen (Erkenntnis-)Wert haben, nicht von einem Mitgliedstaat aufgrund eines archäologischen Interesses unter Schutz gestellt werden können. Die VO Nr. 116/2009 soll jedoch, wie ausgeführt, nur der Durchsetzung solcher Schutzmaßnahmen eines Mitgliedstaats dienen, nämlich sicherstellen, dass 
diese an den Außengrenzen der Union beachtet und eine mit ihnen nicht vereinbare Ausfuhr als nationale Kulturgüter schutzbedürftiger Gegenstände verhindert wird.

Allerdings kann entgegen der Ansicht des Klägers weder aus der ausdrücklichen Benennung der "Sammlungen von ... numismatischem Wert" in Anh. I.A Nr. 13 Buchst. b VO Nr. 116/2009 der Umkehrschluss gezogen werden, einzelne Münzen stünden nicht unter dem Schutz der Verordnung, noch vermag der Senat von vornherein auszuschließen, dass einzelnen Münzen oder Medaillen unter Umständen ein archäologischer Wert zukommen mag, der sie als einen „archäologischen Gegenstand“ im Sinne der VO Nr. 116/2009 erscheinen lässt.

Mit Recht macht der Kläger indes sinngemäß geltend, dass Münzen, die aus der sog. Antike stammen, in der Regel keinen solchen archäologischen Wert haben und deshalb keine archäologischen Gegenstände sind, insbesondere wenn es sie in großer Anzahl gibt und sie - worauf auch das Badische Landesmuseum in seiner vom HZA in der mündlichen Verhandlung vorgelegten Stellungnahme hingewiesen hat - nicht (mehr) einem bestimmten Fundort zugeordnet werden können.

Das archäologische Interesse an einem Gegenstand ist in diesem Zusammenhang vom HZA bzw. dem Tatrichter nach den Umständen des Einzelfalls zu bewerten, wobei als wichtige Beurteilungskriterien insbesondere in Betracht kommen, wie der betreffende Gegenstand im Handel bewertet wird und ob gleiche oder vergleichbare Gegenstände in größerem Umfang Gegenstand eines Handels sind, an dem nicht Archäologen bzw. archäologische Institutionen und Sammlungen, sondern Sammler teilnehmen, die solche Münzen nicht aus einem „archäologischen" Interesse, sondern aus Sammelleidenschaft, wegen des ästhetischen Werts der betreffenden Objekte oder anderer Interessen erwerben.

2. Das FG hat - von seinem unzutreffenden rechtlichen Ausgangspunkt aus - keinerlei Feststellungen dazu getroffen, dass die streitgegenständlichen Münzen und Medaillen aus irgendwelchen besonderen Gründen eine Bedeutung und irgendeinen Wert für die Archäologie hätten. Auch das HZA hat dazu nichts beigetragen. Die ihm vom Landesmuseum Württemberg unter dem 28. April 2009 erteilte Auskunft wäre keine geeignete Grundlage für eine entsprechende Feststellung, zumal sie widersprüchlich ist, wenn einerseits sinngemäß die Erteilung einer Ausfuhrgenehmigung für erforderlich erklärt wird, andererseits das Landesmuseum offenlässt, ob es sich überhaupt um „archäologisches Gut" handelt.

Hingegen hat der Kläger unwidersprochen vorgetragen, es handele sich um "Massenware“, wie sie sich vielfach im allgemeinen Handel befinde. Davon kann der erkennende Senat bei seiner Entscheidung ausgehen, da nichts dafür erkennbar ist, dass das FG, dem allerdings grundsätzlich die tatsächliche Aufklärung und Bewertung solcher Gegebenheiten obliegt, bei einer Zurückverweisung der Sache gemäß § 126 Abs. 3 Satz 1 Nr. 2 FGO in einem zweiten Rechtsgang zu anderen Erkenntnissen kommen könnte.

Da das FG die vom Kläger zur Ausfuhr gestellten Objekte nach alledem zu Unrecht als archäologische Gegenstände angesehen hat, ist sein Urteil aufzuheben. Die Sache ist spruchreif. Der Kläger hat Anspruch auf Annahme seiner Ausfuhranmeldung, ohne dass er dafür eine Ausfuhrgenehmigung vorlegen muss. (Entscheidung von der Redaktion bearbeitet.) 